Discrete Comput Geom 26:73-87 (2001)

DOI: $10.1007 / \mathrm{s} 00454-001-0004-4$

\title{
Grid Drawings of 4-Connected Plane Graphs
}

\author{
K. Miura, ${ }^{1}$ S. Nakano, ${ }^{2}$ and T. Nishizeki ${ }^{1}$ \\ ${ }^{1}$ Graduate School of Information Sciences, Tohoku University, \\ Aoba-yama 05, Sendai 980-8579, Japan \\ \{miura, nishi\}@nishizeki.ecei.tohoku.ac.jp \\ ${ }^{2}$ Department of Computer Science, Faculty of Engineering, Gunma University, \\ 1-5-1 Tenjin-cho, Kiryu, Gunma 376-8515, Japan \\ nakano@cs.gunma-u.ac.jp
}

\begin{abstract}
A grid drawing of a plane graph $G$ is a drawing of $G$ on the plane so that all vertices of $G$ are put on plane grid points and all edges are drawn as straight line segments between their endpoints without any edge-intersection. In this paper we give a very simple algorithm to find a grid drawing of any given 4-connected plane graph $G$ with four or more vertices on the outer face. The algorithm takes time $O(n)$ and yields a drawing in a rectangular grid of width $\lceil n / 2\rceil-1$ and height $\lfloor n / 2\rfloor$ if $G$ has $n$ vertices. The algorithm is best possible in the sense that there are an infinite number of 4-connected plane graphs, any grid drawings of which need rectangular grids of width $\lceil n / 2\rceil-1$ and height $\lfloor n / 2\rfloor$.
\end{abstract}

\section{Introduction}

Recently automatic aesthetic drawing of graphs has created intense interest due to their broad applications, and as a consequence, a number of drawing methods have appeared [1]-[11], [14], [16]. The most typical method is the straight line drawing in which all edges of a graph are drawn as straight line segments without any edge-intersection. Every plane graph has a straight line drawing [8], [15], [17]. However, not every straight line drawing is an aesthetic drawing since many vertices may be concentrated in a small area.

A straight line drawing of a plane graph $G$ is called a grid drawing of $G$ if the vertices of $G$ are put on grid points of integer coordinates. Of course, the distance between any two vertices in the drawing is at least 1 . The integer grid of size $W \times H$ consists of $W+1$ vertical segments and $H+1$ horizontal segments, and has a rectangular contour. $W$ and $H$ are called the width and height of the integer grid, respectively. It is known that every plane graph of $n \geq 3$ vertices has a grid drawing on an $(n-2) \times(n-2)$ 


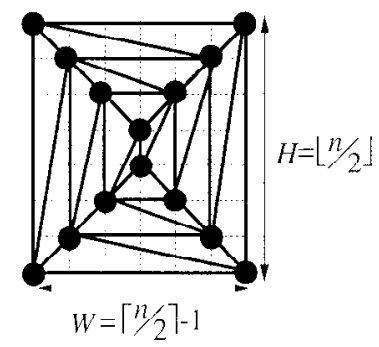

Fig. 1. Nested quadrangles attaining our bounds.

grid, and that such a grid drawing can be found in linear time [5], [7], [9], [14]. It is also shown that, for each $n \geq 3$, there exists a plane graph which needs a grid of size at least $\lfloor 2(n-1) / 3\rfloor \times\lfloor 2(n-1) / 3\rfloor$ for any grid drawing [6], [9]. It has been conjectured that every plane graph has a grid drawing on a $\lceil 2 n / 3\rceil \times\lceil 2 n / 3\rceil$ grid, but this is still an open problem. On the other hand, a restricted class of graphs has a more compact grid drawing. For example, if $G$ is a 4-connected plane graph and has at least four vertices on its outer face, then $G$ has a grid drawing on a $W \times H$ grid such that $W \leq(n+3) / 2, H \leq 2(n-1) / 3$, and $W+H \leq n$, and one can find such a grid drawing in linear time [10]. However, the algorithm is rather complicated.

In this paper we give a very simple algorithm which finds a grid drawing of any given 4-connected plane graph $G$ on a $W \times H$ grid such that $W=\lceil n / 2\rceil-1$ and $H=\lfloor n / 2\rfloor$ in linear time if $G$ has four or more vertices on the outer face. Since $W=\lceil n / 2\rceil-1$ and $H=\lfloor n / 2\rfloor, W+H \leq n-1$. Thus all our bounds on $W, H$, and $W+H$ are better than He's bounds [10]. Our bounds are indeed best possible, because there exist an infinite number of 4-connected plane graphs, for example, the nested quadrangles depicted in Fig. 1, which need grids of size at least $W=\lceil n / 2\rceil-1$ and $H=\lfloor n / 2\rfloor$ for any grid drawing. An aspect ratio of a drawing obtained by the algorithm [10] may be $1: 2$, while the ratio of our algorithm is always $1: 1$. Both our algorithm and the proof of its correctness are very simple.

The outline of our algorithm is as follows. One can assume without loss of generality that a given graph $G$ is internally triangulated as illustrated in Fig. 2(a). First, we find a "4-canonical ordering" of $G$ [12]. Using the ordering, we then divide $G$ into two graphs $G^{\prime}$ and $G^{\prime \prime}$, each of which has about $n / 2$ vertices as illustrated in Fig. 2(b) where $G^{\prime}$ and $G^{\prime \prime}$ are shaded. Next, we draw the plane subgraph $G^{\prime}$ in an isosceles right-angled triangle $\triangle^{\prime}=A^{\prime} B^{\prime} C^{\prime}$ whose base $B^{\prime} C^{\prime}$ has length $W^{\prime}=n / 2-1$ and whose height is $H^{\prime}=W^{\prime} / 2$, as illustrated in Fig. 2(c). Similarly, we draw $G^{\prime \prime}$ in a congruent triangle $\triangle^{\prime \prime}=A^{\prime \prime} B^{\prime \prime} C^{\prime \prime}$ with its base upside down. In Fig. 2(c) the two triangles are drawn by thick hatched lines. We place the two triangles so that their vertices $A^{\prime}$ and $A^{\prime \prime}$ opposite to their bases are separated by distance 1 . Finally, we combine the drawings of $G^{\prime}$ and $G^{\prime \prime}$ to obtain a grid drawing of $G$, as illustrated in Fig. 2(d). The drawings of $G$ has sizes $W=W^{\prime}=n / 2-1$ and $H=2 H^{\prime}+1=W^{\prime}+1=n / 2$.

The remainder of the paper is organized as follows. In Section 2 we give some definitions and lemmas, and present an algorithm to draw $G$ in a grid of $W=\lceil n / 2\rceil-1$ 


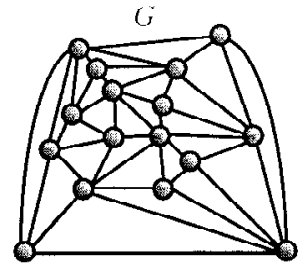

(a)

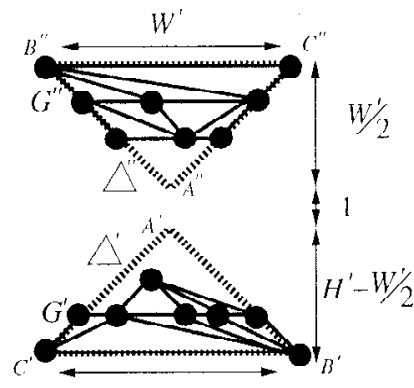

$W^{\prime}-11 / 2-1$

(c)

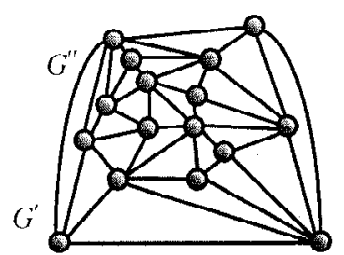

(b)

Fig. 2. Drawing process of our algorithm.

and $H=\lceil n / 2\rceil$. In Section 3 we show how to draw $G^{\prime}$ and $G^{\prime \prime}$. In Section 4 we show that the height $H=\lceil n / 2\rceil$ can be slightly improved to $H=\lfloor n / 2\rfloor$. Finally we conclude in Section 5. An early version of the paper was presented in [13].

\section{Algorithm}

In this section we first introduce some definitions and lemmas, and then present our algorithm and a theorem.

Let $G=(V, E)$ be a simple connected graph, which has no multiple edges or loops. $V$ is the vertex set and $E$ is the edge set of $G$. Let $n$ be the number of vertices of $G$. An edge joining vertices $u$ and $v$ is denoted by $(u, v)$. The degree of a vertex $v$ in $G$ is the number of neighbors of $v$ in $G$, and is denoted by $d(v, G)$. The connectivity $\kappa(G)$ of a graph $G$ is the minimum number of vertices whose removal results in a disconnected graph or a single-vertex graph $K_{1}$. A graph $G$ is $k$-connected if $\kappa(G) \geq k$.

A graph is planar if it can be embedded in the plane so that no two edges intersect geometrically except at a vertex to which they are both incident. A plane graph is a planar graph with a fixed embedding. A plane graph divides the plane into connected regions called faces. We denote the boundary of a face by a clockwise sequence formed by the vertices and edges on the boundary. We call the boundary of the outer face of a plane graph $G$ the contour of $G$, and denote it by $C_{\mathrm{o}}(G)$. A plane graph $G$ is internally triangulated if all inner faces of $G$ are triangles. We can assume without loss of generality that a given graph $G$ is internally triangulated. Otherwise, we internally triangulate $G$ by 


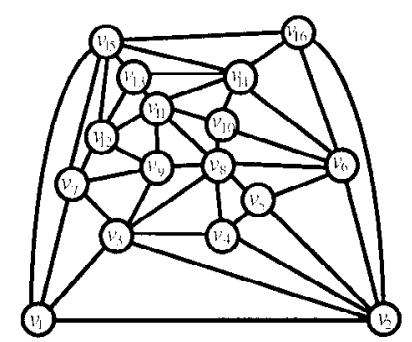

(a)

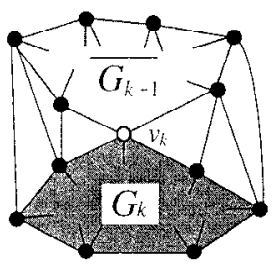

(b)

Fig. 3. (a) A 4-canonical ordering of a 4-connected plane graph of $n=16$ vertices, and (b) an illustration for the condition (co2).

adding some new edges to $G$, find a drawing of the resulting graph, and finally remove the added edges to obtain a drawing of $G$.

We then give a definition of a 4-canonical ordering of a plane graph $G$ [12], on which both our algorithm and He's [10] are based. The 4-canonical ordering is a generalization of the "canonical ordering" [9] which is used to find a grid drawing of triangulated plane graph. Let $\Pi=\left(v_{1}, v_{2}, \ldots, v_{n}\right)$ be an ordering of set $V$. Figure 3(a) illustrates an ordering of the graph $G$ in Fig. 2(a). Let $G_{k}, 1 \leq k \leq n$, be the plane subgraph of $G$ induced by the vertices in $\left\{v_{1}, v_{2}, \ldots, v_{k}\right\}$, and let $\overline{G_{k}}$ be the plane subgraph of $G$ induced by the vertices in $\left\{v_{k+1}, v_{k+2}, \ldots, v_{n}\right\}$. Thus $G=G_{n}=\overline{G_{0}}$. In Fig. 3(b), $G_{k}$ is darkly shaded, while $\overline{G_{k-1}}$ is lightly shaded. We say that $\Pi$ is a 4-canonical ordering of $G$ if the following two conditions are satisfied:

(co1) $v_{1}$ and $v_{2}$ are the ends of an edge on $C_{0}(G)$, and $v_{n}$ and $v_{n-1}$ are the ends of another edge on $C_{\mathrm{o}}(G)$; and

(co2) for each $k, 3 \leq k \leq n-2, v_{k}$ is on $C_{\mathrm{o}}\left(G_{k}\right), d\left(v_{k}, G_{k}\right) \geq 2$, and $d\left(v_{k}, \overline{G_{k-1}}\right)$ $\geq 2$.

Although the definition of a 4-canonical ordering above is slightly different from that in [12], they are effectively equivalent to each other. The following lemma is known.

Lemma 1 [12]. Let $G$ be a 4-connected plane graph having at least four vertices on $C_{\mathrm{o}}(G)$. Then $G$ has a 4 -canonical ordering $\Pi$, and $\Pi$ can be found in linear time.

We are now ready to present our algorithm Draw.

\section{Procedure Draw $(G)$}

\section{begin}

1 Find a 4-canonical ordering $\Pi=\left(v_{1}, v_{2}, \ldots, v_{n}\right)$ of a given 4-connected plane graph $G=(V, E)$;

2 Divide $G$ into two subgraphs $G^{\prime}$ and $G^{\prime \prime}$ where $n^{\prime}=\lceil n / 2\rceil, G^{\prime}=G_{n^{\prime}}$, and $G^{\prime \prime}=\overline{G_{n^{\prime}}}$;

3 Draw $G^{\prime}$ in an isosceles right-angled triangle $\triangle^{\prime}=A^{\prime} B^{\prime} C^{\prime}$ whose base $B^{\prime} C^{\prime}$ has length $W^{\prime}=\lceil n / 2\rceil-1$ and whose height is $H^{\prime}=W^{\prime} / 2$; 
4 Draw $G^{\prime \prime}$ in a congruent triangle $\triangle^{\prime \prime}=A^{\prime \prime} B^{\prime \prime} C^{\prime \prime}$ with its base upside down;

5 Place the two triangles so that their vertices $A^{\prime}$ and $A^{\prime \prime}$ opposite to their bases have the same $x$-coordinate and are separated by distance 1 and that all vertices in two drawings are placed on grid points;

6 Draw every edge of $G$ joining a vertex in $G^{\prime}$ and a vertex in $G^{\prime \prime}$ by a straight line segment;

end.

We say that a curve in the plane is $x$-monotone if the intersection of the curve and any vertical line is empty or a single point. We then have the following lemma for the drawing of $G^{\prime}$, the proof of which is given later in Section 3 .

Lemma 2. One can find in linear time a grid drawing of $G^{\prime}$ satisfying the following conditions:

(a) the drawing is in an isosceles right-angled triangle $\triangle^{\prime}=A^{\prime} B^{\prime} C^{\prime}$ whose base $B^{\prime} C^{\prime}$ has length $W^{\prime}=\lceil n / 2\rceil-1$ and whose height is $H^{\prime}=W^{\prime} / 2$;

(b) the absolute value of the slope of every edge on $C_{\mathrm{o}}\left(G^{\prime}\right)$ is at most 1 ; and

(c) the drawing of the path going clockwise on $C_{\mathrm{o}}\left(G^{\prime}\right)$ from $v_{1}$ to $v_{2}$ is $x$-monotone.

Since $\Pi=\left(v_{1}, v_{2}, \ldots, v_{n}\right)$ is a 4-canonical ordering, the reversed ordering $\Pi^{\prime}=$ $\left(v_{n}, v_{n-1}, \ldots, v_{1}\right)$ is also a 4 -canonical ordering. Therefore $G^{\prime \prime}$ has a grid drawing in a triangle $\triangle^{\prime \prime}$ congruent with $\triangle^{\prime}$. Hence we have the following theorem.

Theorem 1. Algorithm Draw finds in linear time a grid drawing of a given 4-connected plane graph $G$ on $a W \times H$ grid such that $W=\lceil n / 2\rceil-1$ and $H=W+1=\lceil n / 2\rceil$ if there are four or more vertices on the contour $C_{\mathrm{o}}(G)$, i.e., the boundary of the outer face of $G$.

Proof. If step 6 in Draw does not introduce any edge-intersection, then Algorithm Draw correctly finds a grid drawing of $G$ and clearly the size of a drawing of $G$ satisfies $W=W^{\prime}$ and $H=H^{\prime}+H^{\prime}+1$. (See Fig. 2.) By Lemma 2(a) $W^{\prime}=\lceil n / 2\rceil-1$ and $H^{\prime}=W^{\prime} / 2$. Therefore $W=\lceil n / 2\rceil-1$ and $H=W+1=\lceil n / 2\rceil$. Thus we shall show that step 6 does not introduce any edge-intersection.

An oblique side of each isosceles right-angled triangle has slope +1 and the other oblique side has slope -1 . The two vertices $A^{\prime}$ and $A^{\prime \prime}$ have the same $x$-coordinate and are separated by distance 1 : if $A^{\prime}$ has a coordinate $(x, y)$, then $A^{\prime \prime}$ has a coordinate $(x, y+1)$. Therefore the absolute value of the slope of any straight line connecting a point in $\Delta^{\prime}$ and a point in $\Delta^{\prime \prime}$ is greater than the slope $H / W=1+1 / W(>1)$ of a diagonal of the $W \times H$ rectangle. Thus, the absolute value of the slope of any edge connecting a vertex on $C_{\mathrm{o}}\left(G^{\prime}\right)$ and a vertex on $C_{\mathrm{o}}\left(G^{\prime \prime}\right)$ is greater than 1. (See Fig. 4.) On the other hand, by Lemma 2(b) the absolute value of the slope of every edge on $C_{\mathrm{o}}\left(G^{\prime}\right)$ or $C_{\mathrm{o}}\left(G^{\prime \prime}\right)$ is less than or equal to 1 . Furthermore, by Lemma 2(c) both the drawing of the path from $v_{1}$ to $v_{2}$ on $C_{\mathrm{o}}\left(G^{\prime}\right)$ and the drawing of the path from $v_{n-1}$ to $v_{n}$ on $C_{0}\left(G^{\prime \prime}\right)$ are $x$-monotone. Therefore, the straight line drawing of any edge of $G$ connecting a vertex on $C_{\mathrm{o}}\left(G^{\prime}\right)$ and a vertex on 


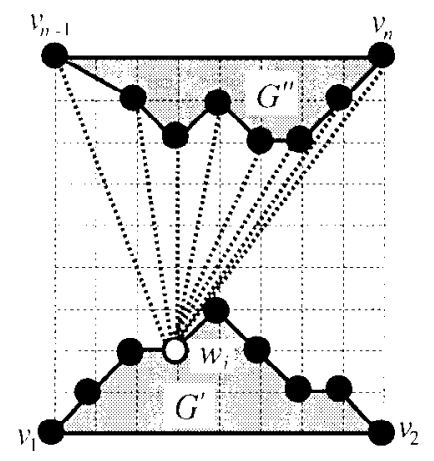

Fig. 4. Illustration for the proof of Theorem 1.

$C_{\mathrm{o}}\left(G^{\prime \prime}\right)$ does not intersect the drawings of $G^{\prime}$ and $G^{\prime \prime}$. Furthermore, the drawings of all these edges do not intersect each other since $G$ is a plane graph and the drawings of the two paths above are $x$-monotone. Thus step 6 does not introduce any edge-intersection.

By Lemma 1 one can execute steps 1 and 2 of procedure Draw in linear time. By Lemma 2 one can execute steps 3 and 4 in linear time. Clearly, one can execute steps 5 and 6 in linear time. Thus Draw runs in linear time.

\section{Drawing $G^{\prime}$}

In this section we show how to find a drawing of $G^{\prime}$ satisfying conditions (a)-(c) in Lemma 2. It suffices to decide only the coordinates of all vertices of $G^{\prime}$, because one can immediately find a straight line drawing from the coordinates.

We first define some terms. Let $x(v)$ and $y(v)$ be the $x$-and $y$-coordinates of vertex $v \in V$, respectively. Let $\Pi=\left(v_{1}, v_{2}, \ldots, v_{n}\right)$ be a 4-canonical ordering of $G$. For any vertices $v_{i}, v_{j} \in V$, we write $v_{i} \prec v_{j}$ iff $1 \leq i<j \leq n$, and write $v_{i} \preceq v_{j}$ iff $1 \leq i \leq j \leq n$. We show later that the following lemma holds.

Lemma 3. If $(u, v)$ is an edge in $G^{\prime}$ and $u \preceq v$, then the $y$-coordinates of vertices $u$ and $v$ decided by our algorithm satisfy $y(u) \leq y(v)$.

We say that a vertex $u$ in a graph $G$ is a smaller neighbor of $v$ if $u$ is a neighbor of $v$ and $u$ is smaller than $v$, that is, $u \prec v$. Similarly, we say that $u$ is a larger neighbor of $v$ if $u$ is a neighbor of $v$ and $u \succ v$. The smallest one among the neighbors of vertex $v$ is called the smallest neighbor of $v$, and is denoted by $w_{s}(v)$. We often denote $w_{s}(v)$ simply by $w_{s}$. The definition of a 4-canonical ordering implies that each vertex $v_{k}, 3 \leq k \leq n-2$, has at least two smaller neighbors and at least two larger neighbors. Let $3 \leq k \leq n$, and let $C_{\mathrm{o}}\left(G_{k-1}\right)=w_{1}, w_{2}, \ldots, w_{m}$, where $w_{1}=v_{1}$ and $w_{m}=v_{2}$. Since $G$ is internally triangulated, all the smaller neighbors of $v_{k}$ consecutively appear on $C_{\mathrm{o}}\left(G_{k-1}\right)$. Thus one may assume that they are $w_{l}, w_{l+1}, \ldots, w_{r}$ for some $l$ and $r, 1 \leq l<r \leq m$, as illustrated in Fig. 5. Furthermore, both all the smaller neighbors of $v_{k}$ and all the larger neighbors of $v_{k}$ consecutively appear in the cyclic adjacent list of $v_{k}$. (See Fig. 3(b).) 


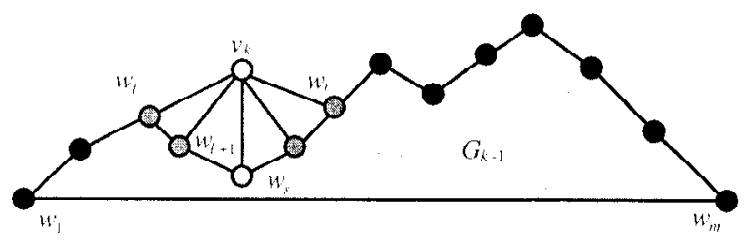

Fig. 5. Illustration of Lemma 4.

We now have the following lemma.

Lemma 4. Let $\Pi=\left(v_{1}, v_{2}, \ldots, v_{n}\right)$ be a 4-canonical ordering of $G$, and let $w_{l}, w_{l+1}$, $\ldots, w_{r}$ be the smaller neighbors of $v_{k}, 3 \leq k \leq n$. Then the following hold:

(a) there is no index $t$ such that $l<t<r$ and $w_{t-1} \prec w_{t} \succ w_{t+1}$; and

(b) $w_{l} \succeq w_{l+1} \succeq \cdots \succeq w_{\mathrm{s}} \preceq \cdots \preceq w_{r}$, and $y\left(w_{l}\right) \geq y\left(w_{l+1}\right) \geq \cdots \geq y\left(w_{\mathrm{s}}\right) \leq$ $\cdots \leq y\left(w_{r}\right)$ where $w_{s}=w_{s}\left(v_{k}\right)$. (See Fig. 5.)

Proof. (a) A proof of (a) is sketched in Lemma 6 of [10] but we provide a more detailed proof here. Assume for a contradiction that there is an index $t$ such that $l<t<r$ and $w_{t-1} \prec w_{t} \succ w_{t+1}$. (See Fig. 6.) Let $w_{t}=v_{i}, 1 \leq i \leq k-1$. Since $v_{k}$ is adjacent to $w_{t-1}, w_{t}$ and $w_{t+1}$ in $G_{k}, w_{t}=v_{i}$ is neither on $C_{\mathrm{o}}\left(G_{k}\right)$ nor on $C_{\mathrm{o}}(G)$ and hence $3 \leq i \leq n-2$. Therefore by condition (co2) of the 4-canonical ordering, $w_{t}$ has at least two larger neighbors. Let $v_{j}$ be the largest one among the $w_{t}$ 's neighbors except $v_{k}$. Then $w_{t}=v_{i} \prec v_{j} \neq v_{k}$. Since $w_{t} \prec v_{j}$ and $w_{t-1} \prec w_{t} \succ w_{t+1}$, we have $v_{j} \succ w_{t-1}, w_{t+1}$. Since both all the smaller neighbors of $v_{i}$ and all the larger neighbors of $v_{i}$ consecutively appear in the cyclic adjacency list of $v_{i}$, vertex $v_{j}$ is in the proper inside of either the triangular face $v_{k}, w_{t}, w_{t-1}$ of graph $G_{k}$ or the triangular face $v_{k}, w_{t+1}, w_{t}$, as illustrated in Fig. 6. Since $v_{j}$ is not on $C_{0}(G)$, we have $3 \leq j \leq n-2$. Since $v_{j}$ is not in $G_{k-1}$, we have $v_{k-1} \prec v_{j}$ and hence $v_{k} \prec v_{j}$. Therefore $v_{k}$ is contained in $G_{j}$, and hence $v_{j}$ is not on $C_{\mathrm{o}}\left(G_{j}\right)$, contrary to condition (co2) of the 4-canonical ordering.

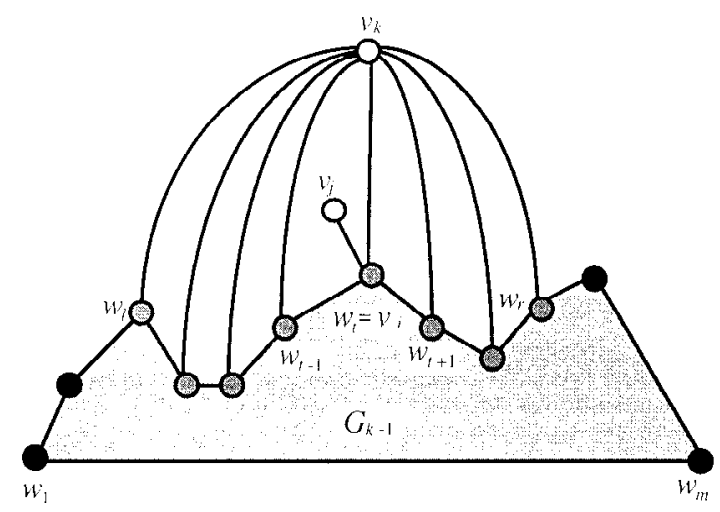

Fig. 6. Graph $G_{k}$ and vertex $v_{j}$. 


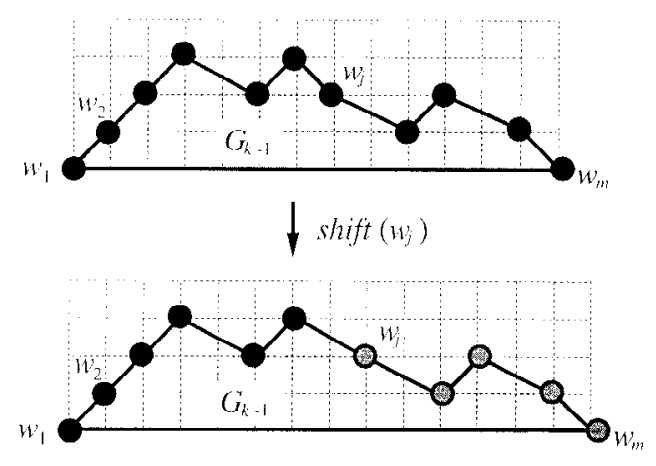

Fig. 7. Shift operation.

(b) Since $w_{s} \preceq w_{r}$, by (a) we have $w_{s} \preceq w_{s+1} \preceq \cdots \preceq w_{r}$. Therefore by Lemma 3 we have $y\left(w_{s}\right) \leq y\left(w_{s+1}\right) \leq \cdots \leq y\left(w_{r}\right)$. Similarly we have $y\left(w_{l}\right) \geq y\left(w_{l+1}\right) \geq \cdots \geq$ $y\left(w_{s}\right)$.

We are now ready to show how to find a drawing of $G^{\prime}$. First, we put vertices $v_{1}, v_{2}, v_{3}$ on grid points $(0,0),(2,0)$, and $(1,1)$ so that $G_{3}$ is drawn as an isosceles right-angled triangle. Clearly, conditions (b) and (c) in Lemma 2 hold for $G_{3}$. Next, for each $k$, $4 \leq k \leq\lceil n / 2\rceil$, we decide the $x$-coordinate $x\left(v_{k}\right)$ and the $y$-coordinate $y\left(v_{k}\right)$ of $v_{k}$ so that conditions (b) and (c) in Lemma 2 hold for $G_{k}$. One may assume that the conditions hold for $G_{k-1}$. Let $C_{\mathrm{o}}\left(G_{k-1}\right)=w_{1}, w_{2}, \ldots, w_{m}$, and let $w_{l}, w_{l+1}, \ldots, w_{r}$ be the smaller neighbors of $v_{k}$. Since condition (c) of Lemma 2 holds for $G_{k-1}$, the drawing of the path $w_{l}, w_{l+1}, \ldots, w_{r}$ is $x$-monotone. Furthermore, by Lemma 4(b), we have $y\left(w_{l}\right) \geq$ $y\left(w_{l+1}\right) \geq \cdots \geq y\left(w_{s}\right) \leq \cdots \leq y\left(w_{r}\right)$, as illustrated in Figs. 5, 8, and 9 .

We always shift some vertices of $G_{k-1}$ to the $x$-direction before adding vertex $v_{k}$, as illustrated in Fig. 7. This can be done by the so-called shift operation [5]-[7], [9]-[11]. We have to determine which vertices of $G_{k-1}$ must be shifted to the $x$-direction. Thus we maintain a set $U\left(v_{k}\right)$ for each vertex $v_{k}, 1 \leq k \leq\lceil n / 2\rceil$. This set will contain vertices located "under" $v_{k}$ that need to be shifted whenever $v_{k}$ is shifted. Initially, we set $U\left(v_{k}\right)=$ $\left\{v_{k}\right\}$ for $k=1,2,3$. For $k, 4 \leq k \leq\lceil n / 2\rceil$, we set $U\left(v_{k}\right)=\left\{v_{k}\right\} \cup\left(\bigcup_{i=l+1}^{r-1} U\left(w_{i}\right)\right)$. Thus all vertices in $U\left(v_{k}\right)$ except $v_{k}$ are not on $C_{\mathrm{o}}\left(G_{k}\right)$. The shift operation on a vertex $w_{j}$, denoted by $\operatorname{shift}\left(w_{j}\right)$, is achieved by increasing the $x$-coordinate of each vertex $u \in \bigcup_{i=j}^{m} U\left(w_{i}\right)$ by 1 [5]-[7].

We then show how to decide $y\left(v_{k}\right)$ and $x\left(v_{k}\right)$. Let $y_{\max }$ be the maximum value of $y$-coordinates of $w_{l}, w_{l+1}, \ldots, w_{r}$, then either $y_{\max }=y\left(w_{l}\right)$ or $y_{\max }=y\left(w_{r}\right)$. There are the following six cases as illustrated in Fig. 8:

(i) $y\left(w_{l}\right)<y\left(w_{r}\right)=y_{\max }$;

(ii) $y_{\max }=y\left(w_{l}\right)>y\left(w_{r}\right)$;

(iii) $y\left(w_{l}\right)=y\left(w_{r}\right)=y_{\max }, l<s<r$, and $y\left(w_{l+1}\right) \neq y_{\max }$;

(iv) $y\left(w_{l}\right)=y\left(w_{r}\right)=y_{\max }, l<s<r$, and $y\left(w_{l+1}\right)=y_{\max }$;

(v) $y\left(w_{l}\right)=y\left(w_{r}\right)=y_{\max }$ and $s=l$; and

(vi) $y\left(w_{l}\right)=y\left(w_{r}\right)=y_{\max }$ and $s=r$. 

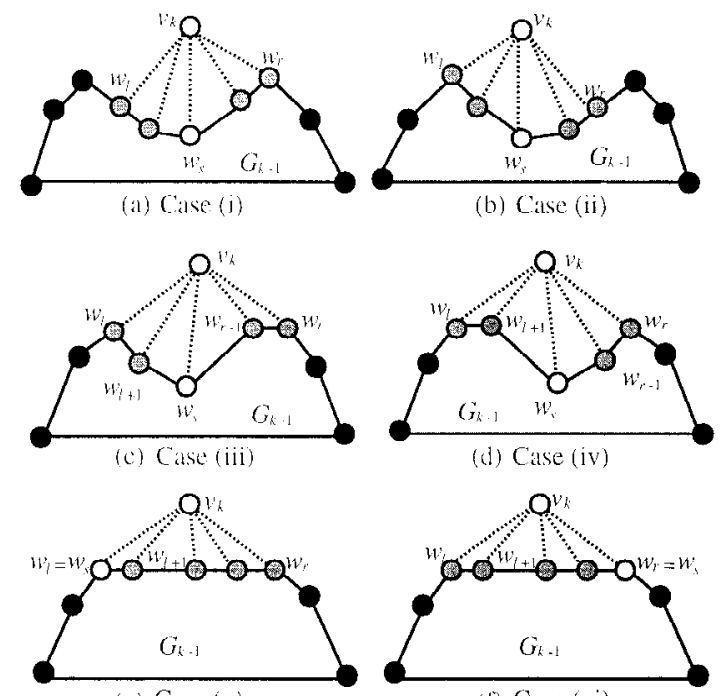

(c) Catse (v)

(1) Cuse (vi

Fig. 8. Illustration of cases (i)-(iv).

We first consider the three cases (i), (iii), and (v), and then consider the remaining three cases (ii), (iv), and (vi).

Cases (i), (iii), and (v). In these cases $y_{\max }=y\left(w_{r}\right)$. We decide $y\left(v_{k}\right)$ and $x\left(v_{k}\right)$ as follows. We first execute shift $\left(w_{s+1}\right)$, that is, we increase the $x$-coordinates of all vertices $w_{s+1}, w_{s+2}, \ldots, w_{m}$ and all vertices under them by 1, as illustrated in Fig. 9(a), (c), and (e). We then decide

$$
y\left(v_{k}\right)= \begin{cases}y_{\max } & \text { if } \quad y\left(w_{r-1}\right)<y_{\max }, \\ y_{\max }+1 & \text { if } \quad y\left(w_{r-1}\right)=y_{\max },\end{cases}
$$

and set $x\left(v_{k}\right)$ so that edge $\left(w_{s}, v_{k}\right)$ has slope 1, i.e.,

$$
x\left(v_{k}\right)=x\left(w_{s}\right)+y\left(v_{k}\right)-y\left(w_{s}\right) .
$$

We denote the slope of a straight line segment $u v$ by slope $(u v)$. Then clearly we have

$$
\operatorname{slope}\left(w_{s} v_{k}\right)=\frac{y\left(v_{k}\right)-y\left(w_{s}\right)}{x\left(v_{k}\right)-x\left(w_{s}\right)}=1 .
$$

Since $y\left(v_{k}\right) \geq y\left(w_{l}\right) \geq y\left(w_{s}\right)$ and $x\left(w_{l}\right) \leq x\left(w_{s}\right)<x\left(v_{k}\right)$, we have

$$
0 \leq \operatorname{slope}\left(w_{l} v_{k}\right)=\frac{y\left(v_{k}\right)-y\left(w_{l}\right)}{x\left(v_{k}\right)-x\left(w_{l}\right)} \leq \operatorname{slope}\left(w_{s} v_{k}\right)=1
$$

as illustrated in Fig. 9(a), (c), and (e).

If $y\left(w_{r-1}\right)<y_{\max }$, then $y\left(v_{k}\right)=y_{\max }=y\left(w_{r}\right)$ and hence slope $\left(v_{k} w_{r}\right)=0$ as illustrated in Fig. 9(a). On the other hand, if $y\left(w_{r-1}\right)=y_{\max }$, then $y\left(v_{k}\right)=y\left(w_{r}\right)+1$, 


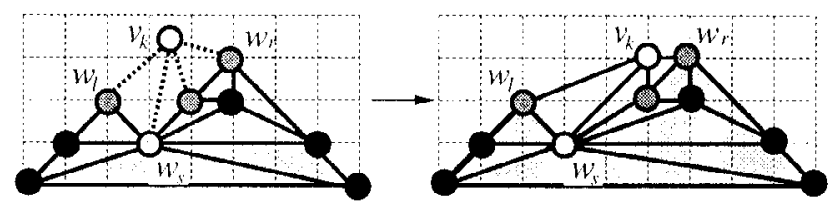

(a) Case (i)

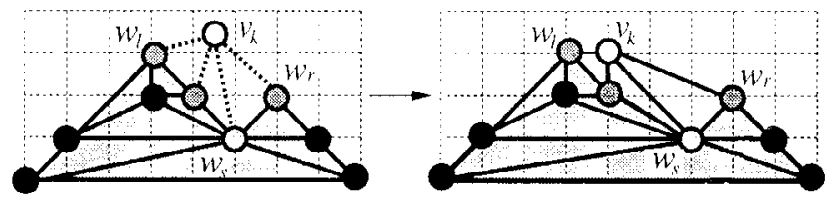

(b) Case (ii)

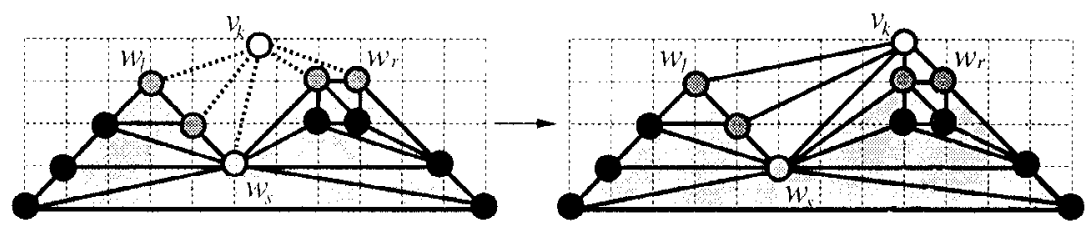

(c) Case (iii)

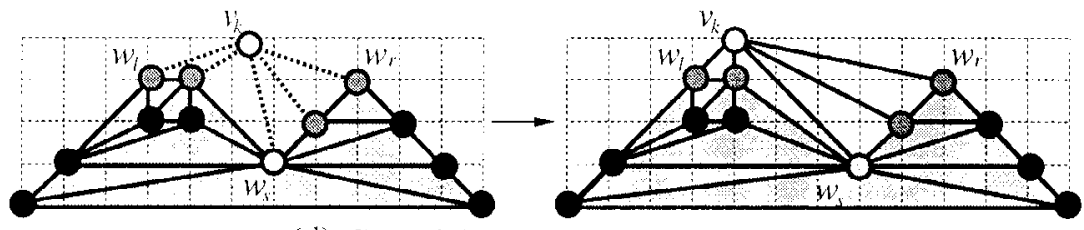

(d) Calse (iv)

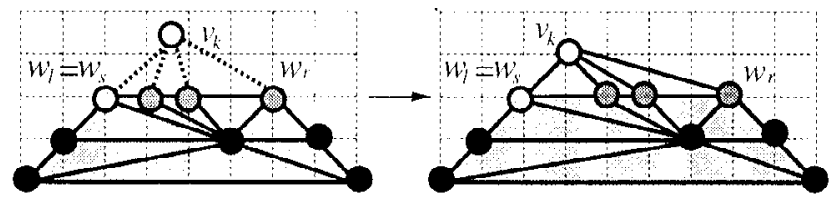

(e) Case (v)

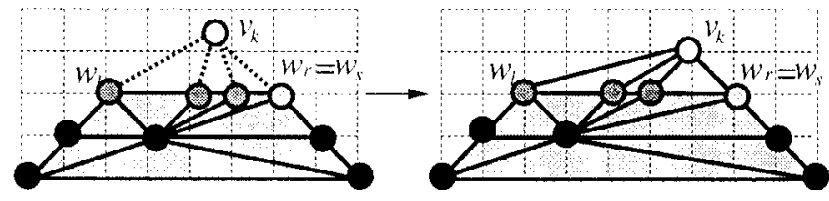

(f) Case (vi)

Fig. 9. How to place $v_{k}$. 
$x\left(v_{k}\right) \leq x\left(w_{r}\right)-1$ and hence we have

$$
-1 \leq \operatorname{slope}\left(v_{k} w_{r}\right)=\frac{y\left(w_{r}\right)-y\left(v_{k}\right)}{x\left(w_{r}\right)-x\left(v_{k}\right)}<0
$$

as illustrated in Fig. 9(c) and (e).

The absolute slope of each straight line segment on $C_{\mathrm{o}}\left(G_{k}\right)$ except $w_{l} v_{k}$ and $v_{k} w_{r}$ is equal to its absolute slope on $C_{\mathrm{o}}\left(G_{k-1}\right)$, and hence is at most 1 .

Thus condition (b) in Lemma 2 holds for $G_{k}$.

One can easily observe that condition (c) in Lemma 2 holds for $G_{k}$.

Cases (ii), (iv), and (vi). In these cases we decide $y\left(v_{k}\right)$ and $x\left(v_{k}\right)$ in a mirror image way of cases (i), (iii), and (v) above. That is, we execute shift $\left(w_{s}\right)$, and decide

$$
y\left(v_{k}\right)=\left\{\begin{array}{lll}
y_{\max } & \text { if } y\left(w_{l+1}\right)<y_{\max }, \\
y_{\max }+1 & \text { if } y\left(w_{l+1}\right)=y_{\max },
\end{array}\right.
$$

and set $x\left(v_{k}\right)$ so that edge $\left(w_{s}, v_{k}\right)$ has slope -1 , i.e.,

$$
x\left(v_{k}\right)=x\left(w_{s}\right)-\left(y\left(v_{k}\right)-y\left(w_{s}\right)\right) .
$$

(See Fig. 9(b), (d), and (f).) Then, similarly as in cases (i), (iii), and (v) above, conditions (b) and (c) hold for $G_{k}$.

Since we decide the $y$-coordinate as above, Lemma 3 clearly holds.

We are now ready to prove Lemma 2.

Proof of Lemma 2. As shown above, conditions (b) and (c) hold. Therefore the absolute value of the slope of every edge on $C_{\mathrm{o}}\left(G^{\prime}\right)$ is at most 1 , and the drawing of the path going clockwise on $C_{\mathrm{o}}(G)$ from $v_{1}$ to $v_{2}$ is $x$-monotone.

The drawing of $G_{3}$ has width 2 . We execute the shift operation once when we add a vertex $v_{k}, 4 \leq k \leq n^{\prime}=\lceil n / 2\rceil$, to the drawing of $G_{k-1}$. Therefore the width $W^{\prime}$ of the drawing of $G^{\prime}$ is $W^{\prime}=2+\left(n^{\prime}-3\right)=\lceil n / 2\rceil-1$. Since conditions (b) and (c) hold, the height is at most $W^{\prime} / 2$. Therefore $G^{\prime}$ is drawn in an isosceles right-angled triangle $\triangle^{\prime}=A^{\prime} B^{\prime} C^{\prime}$ whose base $B^{\prime} C^{\prime}$ has length $W^{\prime}=\lceil n / 2\rceil-1$ and whose height is $H^{\prime}=W^{\prime} / 2$. Thus condition (a) holds.

We then show that the drawing of $G^{\prime}$ obtained by our algorithm is a grid drawing. Our algorithm puts each $v_{k}, 4 \leq k \leq\lceil n / 2\rceil$, on a grid point. Clearly, each edge $\left(v_{k}, w_{j}\right)$, $l \leq j \leq r$, does not intersect any edge of $G_{k-1}$. Furthermore, similarly to the proof of Lemma 2 in [7], one can easily prove by induction on $k$ that any number of executions of the shift operation for $G_{k-1}$ introduce no edge-intersection in $G_{k-1}$. Thus our algorithm obtains a grid drawing of $G^{\prime}$.

All operations in our algorithm except the shift operation can be executed in total time $O(n)$. A simple implement of the shift operation takes time $O(n)$, and our algorithm executes the shift operation at most $\lceil n / 2\rceil$ times. Therefore a straightforward implementation would take time $O\left(n^{2}\right)$. However, using a data structure in [7] to represent the sets $U\left(w_{i}\right), 1 \leq i \leq m$, one can implement the shift operation so that the total time required by the operation is $O(n)$.

Thus our algorithm finds a drawing of $G^{\prime}$ in time $O(n)$. 


\section{Main Theorem}

In this section, modifying step 5 of Algorithm Draw, we slightly improve the bound $H=\lceil n / 2\rceil$ in Theorem 1 to a bound $H=\lfloor n / 2\rfloor$. We may assume without loss of generality that $n$ is an odd integer, because $\lceil n / 2\rceil=\lfloor n / 2\rfloor$ if $n$ is an even integer. The rough idea is as follows. Since $n$ is odd, $n^{\prime \prime}=\lfloor n / 2\rfloor=\lceil n / 2\rceil-1=n^{\prime}-1$ and hence $G^{\prime \prime}$ can be drawn in a triangle $\Delta^{\prime \prime}$ whose height is smaller than that of $\Delta^{\prime}$ for $G^{\prime}$ by $\frac{1}{2}$. Furthermore, one of the tips $A^{\prime}$ and $A^{\prime \prime}$ of the two triangles $\Delta^{\prime}$ and $\Delta^{\prime \prime}$ is not placed on a grid point. As we show in a detailed case analysis, it is then always possible to place $A^{\prime}$ and $A^{\prime \prime}$ at $y$-distance $\frac{1}{2}$ only, and still have a planar grid drawing. Thus one can save one unit in height.

For points $a$ and $b$, we denote by $(a, b]$ the half-open straight line segment connecting $a$ and $b$. Since $n$ is odd, either $n=1(\bmod 4)$ or $n=3(\bmod 4)$. However, we assume that $n=3(\bmod 4)$; the argument for the case $n=1(\bmod 4)$ is similar to one for the case $n=3(\bmod 4)$ except that the roles of $A^{\prime}$ and $A^{\prime \prime}$ are interchanged. Since $G^{\prime}$ has $n^{\prime}=\lceil n / 2\rceil$ vertices, $G^{\prime}$ has a grid drawing in an isosceles right-angled triangle $\Delta^{\prime}=A^{\prime} B^{\prime} C^{\prime}$ whose base $B^{\prime} C^{\prime}$ has length $W^{\prime}=n^{\prime}-1=\lceil n / 2\rceil-1$ and whose height is $H^{\prime}=W^{\prime} / 2$. On the other hand, since $G^{\prime \prime}$ has $n^{\prime \prime}=\lfloor n / 2\rfloor=n^{\prime}-1$ vertices, $G^{\prime \prime}$ has a grid drawing in an isosceles right-angled triangle $\Delta^{\prime \prime}=A^{\prime \prime} B^{\prime \prime} C^{\prime \prime}$ which is smaller than $\Delta^{\prime}$; the base $B^{\prime \prime} C^{\prime \prime}$ of $\Delta^{\prime \prime}$ has length $W^{\prime \prime}=n^{\prime \prime}-1=\lfloor n / 2\rfloor-1$, and the height of $\Delta^{\prime \prime}$ is $H^{\prime \prime}=W^{\prime \prime} / 2$. Since $n=3(\bmod 4), A^{\prime}$ is not a grid point, but $A^{\prime \prime}$ is a grid point as illustrated in Fig. 10. Therefore $G^{\prime}$ has no vertex on $A^{\prime}$, but $G^{\prime \prime}$ may have a vertex on $A^{\prime \prime}$. It suffices to consider the following three cases:

(a) none of the edges of $G$ joins a vertex of $G^{\prime}$ on line $A^{\prime} C^{\prime}$ and a vertex of $G^{\prime \prime}$ on line $A^{\prime \prime} C^{\prime \prime}$;

(b) none of the edges of $G$ joins a vertex of $G^{\prime}$ on line $A^{\prime} B^{\prime}$ and a vertex of $G^{\prime \prime}$ on line $A^{\prime \prime} B^{\prime \prime}$; and

(c) otherwise.

First consider case (a). Let $(x, y)$ be the coordinate of $A^{\prime}$. In this case, not as in step 5 of Draw, we arrange triangles $\Delta^{\prime}$ and $\Delta^{\prime \prime}$ so that $A^{\prime \prime}$ has a coordinate $\left(x+\frac{1}{2}, y+\frac{1}{2}\right)$ as

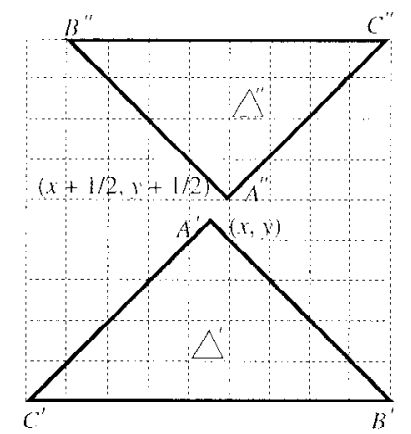

(a)

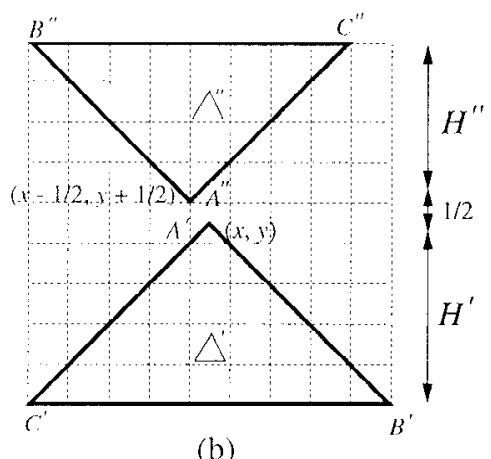

(b)

Fig. 10. Arrangement of triangles $\Delta^{\prime}$ and $\Delta^{\prime \prime}$. 
illustrated in Fig. 10(a). Then $\mid$ slope $(a b) \mid>1$ for any straight line segment $a b$ connecting a point $a$ in $\Delta^{\prime}$ and a point $b$ in $\Delta^{\prime \prime}$ if $a$ is not on line $A^{\prime} C^{\prime}$ or $b$ is not on line $A^{\prime \prime} C^{\prime \prime}$. Therefore the slope slope $(e)$ of the line segment of an edge $e$ satisfies $\mid$ slope $(e) \mid>1$ for every edge $e$ of $G$ joining a vertex in $G^{\prime}$ and a vertex in $G^{\prime \prime}$. Thus no edge-intersection occurs when we draw all these edges by straight line segments in step 6 of Draw.

We next consider case (b). In this case, we arrange triangles $\Delta^{\prime}$ and $\Delta^{\prime \prime}$ so that $A^{\prime \prime}$ has a coordinate $\left(x-\frac{1}{2}, y+\frac{1}{2}\right)$ as illustrated in Fig. 10(b). Then, similarly as in case (a) above, no edge-intersection occurs.

We finally consider case (c). In this case, $G$ has two edges such that one joins a vertex of $G^{\prime}$ on line $\left[A^{\prime}, C^{\prime}\right]$ with a vertex of $G^{\prime \prime}$ on line $\left[A^{\prime \prime}, C^{\prime \prime}\right]$ and the other joins a vertex of $G^{\prime}$ on line $\left[A^{\prime}, B^{\prime}\right]$ with a vertex of $G^{\prime \prime}$ on line $\left[A^{\prime \prime}, B^{\prime \prime}\right]$. Given that the embedded graph $G$ is planar, these criteria can be satisfied only if either $A^{\prime}$ is an endpoint of both edges or $A^{\prime \prime}$ is an endpoint of both edges. However, $A^{\prime}$ is not a grid point and cannot hold a vertex. So $G^{\prime \prime}$ has a vertex $u$ on point $A^{\prime \prime}$ which are incident to both edges. (Indeed $u$ is vertex $v_{n^{\prime}+1}$.) Let $C_{\mathrm{o}}\left(G^{\prime}\right)=w_{1}, w_{2}, \ldots, w_{m}$ where $w_{1}=v_{1}$ and $w_{m}=v_{2}$. Let $w_{l}, w_{l+1}, \ldots, w_{r}$ be the smaller neighbors of $u$ where $1 \leq l<r \leq m$. Then $w_{l}$ must be on line $\left(A^{\prime}, C^{\prime}\right]$, and $w_{r}$ must be on line $\left(A^{\prime}, B^{\prime}\right]$. Let $w_{s}$ be the smallest neighbor of $u$, then by Lemma $4(\mathrm{~b})$ we have $y\left(w_{l}\right) \geq y\left(w_{l+1} \geq \cdots \geq y\left(w_{s}\right) \leq \cdots \leq y\left(w_{r}\right)\right.$. There are the following three cases: (i) $s=r$, (ii) $l<s<r$, and (iii) $s=l$. In cases (i) and (ii), exactly one smaller neighbor $w_{l}$ of $u$ lies on line $\left(A^{\prime}, C^{\prime}\right]$. We arrange $\Delta^{\prime}$ and $\Delta^{\prime \prime}$ so that $A^{\prime \prime}$ has a coordinate $\left(x+\frac{1}{2}, y+\frac{1}{2}\right)$, as illustrated in Fig. 11(a) and (b). Then $\left(u, w_{l}\right)$

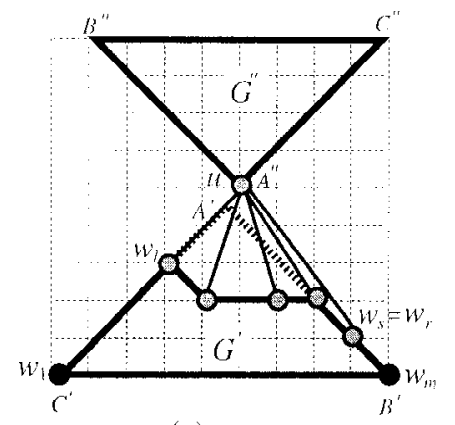

(a) $s=r$

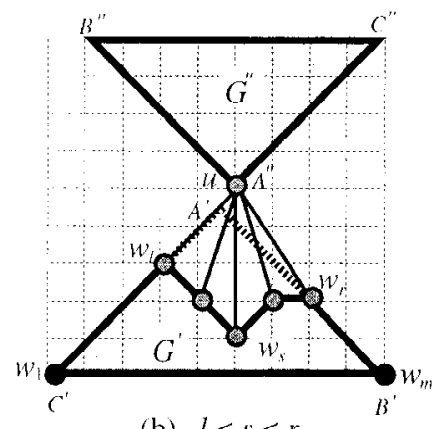

(b) $l<s<r$

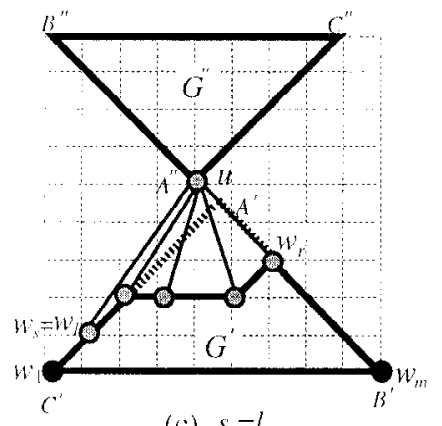

(c) $s=1$

Fig. 11. Drawing for case (c). 
is drawn by a line of slope 1, but $|\operatorname{slope}(e)|>1$ for any other edge $e$ of $G$ joining a vertex in $G^{\prime}$ and a vertex in $G^{\prime \prime}$. Thus no edge-intersection occurs. In case (iii), exactly one smaller neighbor $w_{r}$ of $u$ lies on line $\left(A^{\prime}, B^{\prime}\right]$. We arrange $\Delta^{\prime}$ and $\Delta^{\prime \prime}$ so that $A^{\prime \prime}$ has a coordinate $\left(x-\frac{1}{2}, y+\frac{1}{2}\right)$, as illustrated in Fig. 11(c). Then $\left(u, w_{r}\right)$ is drawn by a line of slope -1 , but $\mid$ slope $(e) \mid>1$ for any other edge $e$ of $G$ joining a vertex in $G^{\prime}$ and a vertex in $G^{\prime \prime}$. Thus no edge-intersection occurs.

In any of the three cases (a), (b), and (c), the height $H$ of a rectangular grid required for $G$ satisfies

$$
H=H^{\prime}+\frac{1}{2}+H^{\prime \prime}=(\lceil n / 2\rceil-1) / 2+\frac{1}{2}+(\lfloor n / 2\rfloor-1) / 2=\lfloor n / 2\rfloor .
$$

Thus we have the following main theorem.

Theorem 2. One can find in linear time a grid drawing of a given 4-connected plane graph $G$ on a $W \times H$ grid such that $W=\lceil n / 2\rceil-1$ and $H=\lfloor n / 2\rfloor$ if there are four or more vertices on $C_{0}(G)$.

\section{Conclusion}

In this paper we give a very simple algorithm which finds a grid drawing of any given 4-connected plane graph $G$ on a $W \times H$ rectangular grid such that $W=\lceil n / 2\rceil-1$ and $H=\lfloor n / 2\rfloor$ in linear time if $G$ has $n$ vertices and four or more vertices are on the outer face. Our algorithm is best possible in the sense that there are an infinite number of 4-connected plane graphs any grid drawings of which need grids of size at least $W=\lceil n / 2\rceil-1$ and $H=\lfloor n / 2\rfloor$. Both the algorithm in [10] and ours are based on the 4-canonical ordering. Our algorithm improves on the algorithm in [10] not only with respect to the area of the drawing, but also with respect to simplicity. Our algorithm requires only six cases for placing a vertex, while the algorithm in [10] requires ten cases. Also, our cases are easily determined by testing very simple conditions on coordinates and the canonical ordering. Furthermore, we introduced a new idea: (1) divide a given plane graph $G$ into two subgraphs; (2) draw each of them in an isosceles right-angled triangle; and (3) obtain a grid drawing of $G$ by combining the drawings of the two subgraphs.

\section{Acknowledgments}

We thank Dr. Xiao Zhou for valuable discussion, and the anonymous referees for their valuable comments and suggestions for improving the presentation of the paper.

\section{References}

1. G. Di Battista, P. Eades, R. Tamassia, and I. G. Tollis, Automatic graph drawing: an annotated bibliography. Computational Geometry: Theory and Applications, 4 (1994), 235-282.

2. G. Di Battista, P. Eades, R. Tamassia, and I. G. Tollis, Graph Drawing. Prentice-Hall, Englewood Cliffs, NJ, 1998. 
3. N. Chiba, K. Onoguchi, and T. Nishizeki, Drawing planar graphs nicely. Acta Informatica, 22 (1985), 187-201.

4. N. Chiba, T. Yamanouchi, and T. Nishizeki, Linear algorithms for convex drawings of planar graphs. In Progress in Graph Theory, J. A. Bondy and U. S. R. Murty (eds.), pp. 153-173. Academic Press, New York, 1984.

5. M. Chrobak and G. Kant, Convex grid drawings of 3-connected planar graphs. International Journal of Computational Geometry and Applications, 7 (1997), 211-223.

6. M. Chrobak and S. Nakano, Minimum-width grid drawings of plane graphs. Computational Geometry: Theory and Applications, 10 (1998), 29-54.

7. M. Chrobak and T. Payne, A linear-time algorithm for drawing planar graphs on a grid. Information Processing Letters, 54 (1995), 241-246.

8. I. Fáry. On straight lines representation of plane graphs. Acta Scientiarum Mathematicarum (Szeged), 11 (1948), 229-233.

9. H. de Fraysseix, J. Pach, and R. Pollack, How to draw a planar graph on a grid. Combinatorica, 10 (1990), $41-51$.

10. X. He, Grid embedding of 4-connected plane graphs. Discrete \& Computational Geometry, 17 (1997), 339-358.

11. G. Kant, Drawing planar graphs using the canonical ordering. Algorithmica, 16 (1996), 4-32.

12. G. Kant and X. He, Regular edge labeling of 4-connected plane graphs and its applications in graph drawing problems. Theoretical Computer Science, 172 (1997), 175-193.

13. K. Miura, S. Nakano, and T. Nishizeki, Grid drawings of four-connected plane graphs. Proceedings of Graph Drawing '99 (GD '99), Lecture Notes in Computer Science, 1731. Springer-Verlag, Berlin, 1999. pp. $145-154$.

14. W. Schnyder, Embedding planar graphs on the grid. Proceedings of the 1st Annual ACM-SIAM Symposium on Discrete Algorithms, San Francisco, 1990, pp. 138-148.

15. S. K. Stein, Convex maps. Proceedings of the American Mathematical Society, 2 (1951), 464-466.

16. W. T. Tutte, How to draw a graph. Proceedings of the London Mathematical Society, 13 (1963), 743-768.

17. K. Wagner, Bemerkungen zum vierfarbenproblem. Jahresbericht der Deutschen MathematikerVereinigung, 46 (1936), 26-32.

Received October 13, 1999, and in revised form July 18, 2000. Online publication February 26, 2001. 